\title{
Extracts of Bauhinia championii (Benth.) Benth. attenuate the inflammatory response in a rat model of collagen-induced arthritis
}

\author{
WEI XU ${ }^{1}$, MINGQING HUANG ${ }^{1}$, YUQIN ZHANG ${ }^{1}$, HUANG LI $^{1}$, HAIYIN ZHENG $^{2}$, \\ LISHUANG YU ${ }^{1}, \mathrm{KEDAN} \mathrm{CHU}^{1}$, YU LIN ${ }^{1}$ and LIDIAN CHEN ${ }^{3}$ \\ ${ }^{1}$ College of Pharmacy; ${ }^{2}$ College of Integrative Medicine; ${ }^{3}$ College of Rehabilitation Medicine, \\ Fujian University of Traditional Chinese Medicine, Fuzhou, Fujian 350122, P.R. China
}

Received October 1, 2015; Accepted February 23, 2016

DOI: $10.3892 / \mathrm{mmr} .2016 .5070$

\begin{abstract}
Rheumatoid arthritis is considered a serious public health problem, which is commonly treated with traditional Chinese or herbal medicine. The present study evaluated the effects of Bauhinia championii (Benth.) Benth. extraction (BCBE) on a type II collagen-induced arthritis (CIA) rat model. Wistar rats with CIA received either 125 or $500 \mathrm{mg} / \mathrm{kg} \mathrm{BCBE}$, after which, paw swelling was markedly suppressed compared with in the model group. In addition, BCBE significantly ameliorated pathological joint alterations, including synovial hyperplasia, and cartilage and bone destruction. The protein and mRNA expression levels of interleukin (IL)-6, IL-8, tumor necrosis factor- $\alpha$ and nuclear factor- $\kappa \mathrm{B}$ in synovial tissue were determined by immunohistochemical staining, western blot analysis and reverse transcription-polymerase chain reaction. The results demonstrated that the expression levels of these factors were significantly downregulated in the BCBE-treated group compared with in the model group. These results indicated that BCBE may exert an inhibitory effect on the CIA rat model, and its therapeutic potential is associated with its anti-inflammatory action.
\end{abstract}

\section{Introduction}

Rheumatoid arthritis (RA) is a type of systemic chronic inflammatory disorder, the prevalence of which varies

Correspondence to: Professor Wei Xu, College of Pharmacy, Fujian University of Traditional Chinese Medicine, 1 Qiuyang Road, Minhou Shangjie, Fuzhou, Fujian 350122, P.R. China

E-mail: xwfjlab@163.com

Abbreviations: RA, rheumatoid arthritis; CIA, collagen-induced arthritis; BCBE, Bauhinia championii (Benth.) Benth. extraction; TGT, tripterygium glycosides tablet; IL, interleukin; TNF- $\alpha$, tumor necrosis factor- $\alpha$; NF- $\kappa \mathrm{B}$, nuclear factor- $\kappa \mathrm{B}$; RT-PCR, reverse transcription-polymerase chain reaction; $\mathrm{HE}$, hematoxylin and eosin

Key words: Bauhinia championii (Benth.) Benth., IL-6, IL-8, $\mathrm{TNF}-\alpha, \mathrm{NF}-\kappa \mathrm{B}$, rheumatoid arthritis worldwide between 0.5 and $1 \%$ (1). RA is an autoimmune disease that is characterized by synovial inflammation, synovial hyperplasia, neovascularization, and ultimately cartilage and bone damage $(2,3)$. RA has a multi-factorial etiology, which is associated with internal and external factors $(4,5)$, including predisposing genes (6), lifestyle choices (7), infectious agents (8), and occupational exposures (9). Although the molecular pathogenesis of RA remains unclear, recent evidence has suggested that the cytokine network has an important role in the pathogenesis of RA (10). Proinflammatory cytokines, such as interleukin (IL)- 6 and tumor necrosis factor (TNF)- $\alpha$, have a major role in inflammation. TNF- $\alpha$ is an important regulatory factor of the inflammatory and immune response, which can strongly promote inflammation, and is associated with various pathological changes of RA (11). IL-6 is a pleiotropic cytokine, which is plentifully expressed in rheumatoid synovium and may contribute to joint damage (12). IL-8 is a chemokine of the immune system, which serves as a chemical signal that attracts neutrophils to the site of inflammation (13). IL-10 is an anti-inflammatory cytokine, which suppresses TNF- $\alpha$ production and the development of type II collagen-induced arthritis (CIA) in rats (14). Nuclear factor (NF) $-\kappa B$, which is a pleiotropic transcription factor, is inactive in quiescent cells; however, it can promote the transcription of various genes and the release of several cytokines, which may contribute to inflammation once activated. An excessive inflammatory reaction can also damage target cells and tissues.

At present, non-steroidal anti-inflammatory drugs, slow-acting anti-rheumatic drugs, adrenocorticotropic hormone, novel biological agents, and some Chinese drugs are used clinically to treat RA. However, due to the lack of efficacy and undesirable side effects associated with Western medical treatments, an effective and safe therapeutic strategy for the treatment of RA is still required.

Traditional Chinese medicine, or herbal medicine, has an important role in the treatment of RA. Plant-derived natural products dominate the traditional medical systems. Bauhinia championii (Benth.) Benth. is a perennial liana, which belongs to the Bauhinia Leguminosae family. It is widely used to treat many diseases. It has a bitter, acerbic taste, and warm in nature. In addition, it has the ability to expel wind and eliminate dampness, promote blood circulation to relieve pain, invigorate the spleen, and regulate qi (15). It is mainly used 
to treat epigastric pain (16), RA (17), and acute and chronic pain of the lumbar region and leg $(18,19)$. Experimental studies focusing on this herbal remedy are few, particularly those regarding the mechanism of action underlying the treatment of RA. Our previous study indicated that Bauhinia championii (Benth.) Benth. is efficacious in inhibiting paw swelling and inflammation in CIA rats (20).

At present, there are no reports identifying cytokines as the target molecules of Bauhinia championii (Benth.) Benth. in the treatment of RA. Therefore, the present study aimed to evaluate the anti-inflammatory effects of Bauhinia championii (Benth.) Benth. on the CIA rat model. The present study observed paw edema and histological alterations in the CIA rats. Furthermore, the effects of Bauhinia championii (Benth.) Benth. on the production of serum TNF- $\alpha$, IL-8, IL-6 and IL-10, and the protein expression of $N F-\kappa B$ in synovial tissue were investigated, in order to elucidate the mechanism by which Bauhinia championii (Benth.) Benth. exerts its effect on CIA.

\section{Materials and methods}

Plantmaterial. Rattans of Bauhinia championii (Benth.) Benth. were collected in the mountainous woods of Minhou (E119.22, N25.88; Fuzhou, China) in April 2011. Bauhinia championii (Benth.) Benth. was identified by Professor Lu Wei (Fujian University of Traditional Chinese Medicine, Fuzhou, China). A voucher specimen was deposited at the Pharmacy College, Fujian University of Traditional Chinese Medicine. The samples were dried, milled, passed through a stainless steel sieve, and stored until use at $4^{\circ} \mathrm{C}$. The same batch of samples was used throughout the present study.

Animals. A total of 50 specific-pathogen-free Wistar male rats, weighing $200 \pm 20 \mathrm{~g}$, were offered by the Animal Care and Use Committee, Fujian University of Traditional Chinese Medicine. The rats were purchased from Shanghai SLAC Laboratory Animal Co. Ltd. (Shanghai, China) [license no. SCX (hu) 2007-2005]. The rats were housed in an environment containing $55 \pm 5 \%$ humidity, with an ambient temperature $\left(21-23^{\circ} \mathrm{C}\right)$, under a 12 -h light/dark cycle with ad libitum access to food and water. Animal welfare was taken into consideration, in accordance with international ethical guidelines and the National Institutes of Health Guide concerning the Care and Use of Laboratory Animals (21). The study was approved by the ethics committee of Fujian University of Traditional Chinese Medicine.

Preparation of ethyl acetate fractions. Extraction of ethyl acetate fractions was prepared according topreviously described methods (22). The prepared $1.0 \mathrm{~g}$ Bauhinia championii (Benth.) Benth. extraction (BCBE) was dissolved in acetonitrile, and was subjected to high-performance liquid chromatography (HPLC) analysis in order to identify the major peaks (Fig. 1). HPLC analysis was performed on a Waters UPLC system (Waters, Ltd., Hertfordshire, UK). Chromatographic separation was performed at $25^{\circ} \mathrm{C}$ on an Diamonsil C18 $(250 \times 4.6 \mathrm{~mm}, 5 \mu \mathrm{m}$; Beijing Dikma Technology Co., Ltd., Beijing, China). The mobile phases consisted of $0.1 \%$ formic acid in (A) water and (B) acetonitrile with $0.1 \%$ formic acid (both purchased from
Sinopharm Chemical Reagent Co., Ltd., Shanghai, China). The gradient elution program was as follows: 0 Min at (A) 25 and (B) $75 \%, 15 \mathrm{~min}$ at (A) 45 and (B) $55 \%$, and $40 \mathrm{~min}$ at (A and B) 50\%. The flow rate was maintained at $1 \mathrm{ml} / \mathrm{min}$, and the ample volume injected was $10 \mu \mathrm{l}$. The extracted fractions were maintained at $4^{\circ} \mathrm{C}$ until subsequent use.

CIA model generation and BCBE administration. The CIA model was generated according to a previous study (22). A total of 50 Wistar rats were randomly divided into five groups: Normal group, model group, tripterygium glycosides tablet (TGT) group, BCBE low-dose group and BCBE high-dose group ( $\mathrm{n}=10 /$ group). Firstly, $2 \mathrm{mg} / \mathrm{ml}$ bovine type II collagen (Sigma-Aldrich, St. Louis, MO, USA) was emulsified with an equal volume of complete Freund's adjuvant (Sigma-Aldrich) to a final concentration of $0.1 \mathrm{mg} / \mathrm{ml}$. Secondly, in all groups apart from the normal group, the rats were injected with $0.2 \mathrm{mg}$ collagen emulsion into the tail root via intradermal injection. A secondary immunization test was performed 7 days later with similar methods. Drugs were administered intragastrically once a day from the first day after primary immunization. The normal group and model group received $10 \mathrm{ml} / \mathrm{kg} 9.0 \mathrm{~g} / 1$ sodium chloride daily; the BCBE high-dose group received $0.5 \mathrm{~g} / \mathrm{kg} \mathrm{BCBE}$ daily; the $\mathrm{BCBE}$ low-dose group received $0.125 \mathrm{~g} / \mathrm{kg} \mathrm{BCBE}$ daily; and the TGT positive control group received $1 \mathrm{mg} / \mathrm{kg}$ TGT daily (Huangshi Feiyun Pharmaceutical Co., Ltd., Huangshi, China). All animals were treated continuously for 28 days.

Measurement of paw edema. Over the course of the experiment, paw volume was measured using a YLS-7B plethysmometer (Yi Yan Technology Development Co., Ltd., Jinan, China). Swelling was expressed as the volume difference $(\mathrm{ml})$ before and after modeling (23).

Determination of IL-6, IL-8,IL-10 and TNF- $\alpha$ levels. The rats were sacrificed with urethane $(1,500 \mathrm{mg} / \mathrm{kg}$; i.p.; Sinopharm Chemical Reagent Co., Ltd.) and blood samples were collected. The blood samples were centrifuged at $1,500 \mathrm{x} \mathrm{g}$ for $20 \mathrm{~min}$ at $4^{\circ} \mathrm{C}$ in order to obtain the supernatant and serum. Enzyme-linked immunosorbent assay kits were used to measure the serum levels of IL-8 (ER-1578; Beijing Zhongshan Golden Bridge Biotechnology Co., Ltd., Beijing, China), IL-6 (113897105), IL-10 (1388127105) (both purchased from Wuhan Boster Biological Technology, Ltd., Wuhan, China) and TNF- $\alpha$ (AE90301Ra) (Shanghai Lianshuo Biological Technology Co., Ltd., Shanghai, China), according to the manufacturer's protocols.

Histopathological examination. Briefly, right ankles were obtained from the rats and were fixed in $10 \%$ formalin solution for histopathological examination. The samples were then decalcified in $12.5 \%$ ethylenediaminetetraacetic acid $(\mathrm{pH} 7.0)$ for 20 days, dehydrated in ethanol, cleared with dimethyl benzene, and embedded in paraffin blocks. The specimens were then serially cut into $5 \mu \mathrm{m}$ sections, and were stained with hematoxylin and eosin (HE; Beyotime Institute of Biotechnology, Haimen, China) prior to observation under a light microscope (DM4000B, Leica Microsystems, Wetzlar, Germany). 


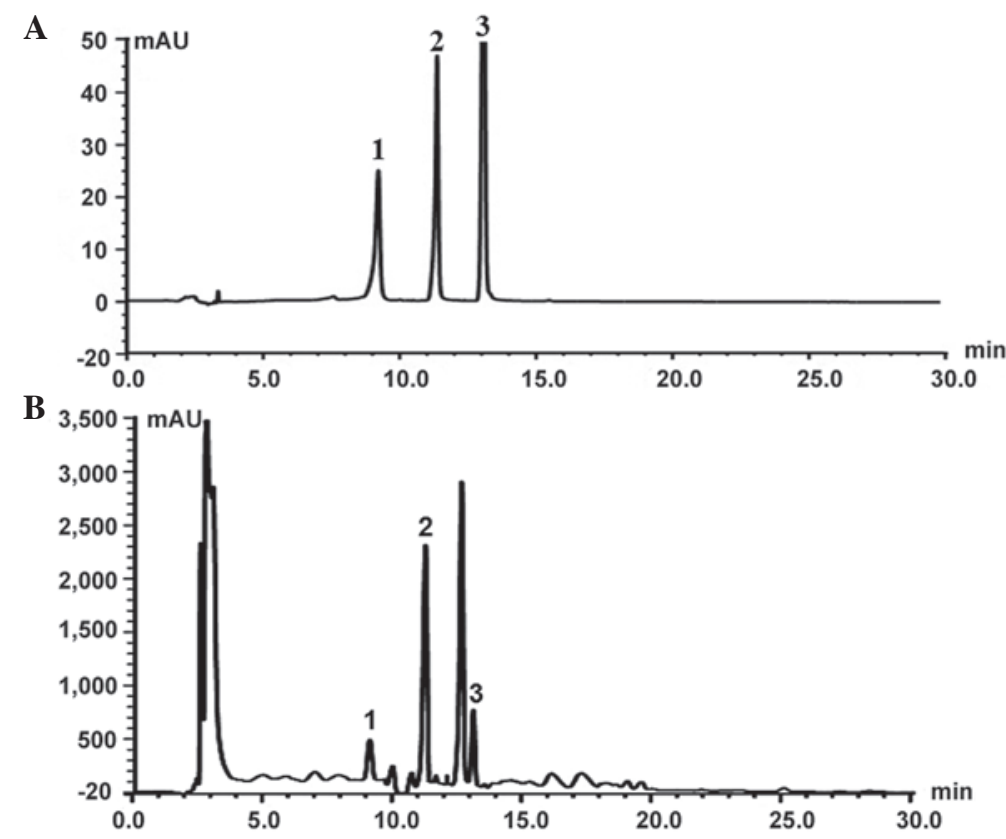

Figure 1. High-performance liquid chromatography profiles of (A) control solution and (B) Bauhinia championii (Benth.) Benth. extraction. Peak 1, myricetin; peak 2, quercetin; peak 3, kaempferol.

Western blot analysis. Synovial tissues were obtained from the hind paws after stripping away the skin, muscle, fatty tissues, bone and tendons. Subsequently, the synovial tissues were immediately frozen in liquid nitrogen and stored at $-80^{\circ} \mathrm{C}$. Randomly, synovial tissues from three rats per group were adequately homogenized using non-denaturing lysis buffer (Beyotime Institute of Biotechnology), and were centrifuged at $12,000 \mathrm{x} \mathrm{g}$ for $15 \mathrm{~min}$ at $4^{\circ} \mathrm{C}$. The protein concentration in the supernatants was then determined using a BCA Protein Assay Kit (Beyotime Institute of Biotechnology). The supernatants were denatured in protein loading buffer, and protein samples $(30 \mu \mathrm{g})$ were separated by $12 \%$ sodium dodecyl sulfate-polyacrylamide gel electrophoresis and were transferred to polyvinylidene difluoride membranes (EMD Millipore, Billerica, MA, USA). Non-specific binding was blocked for $3 \mathrm{~h}$ with $5 \%$ skim milk in Tris-buffered saline containing $0.1 \%$ Tween. Membranes were incubated with rabbit NF- $\kappa$ B p65 (cat. no. 8242) and $\beta$-actin (cat. no. 4967) primary antibodies (1:1,000 dilution; Cell Signaling Technology, Danvers, MA, USA) overnight at $4^{\circ} \mathrm{C}$, and were then incubated with an appropriate horseradish peroxidase-conjugated secondary antibody (1:500 dilution; cat. no. C-0029; Beijing Biosynthesis Biotechnology Co., Ltd., Beijing, China) for $2 \mathrm{~h}$ at room temperature. Subsequently, the membranes were washed and were visualized using enhanced chemiluminescence western detection reagents (Beyotime Institute of Biotechnology).

Immunohistochemical analysis. Paraffin-embedded sections (5 $\mu \mathrm{m})$ were used for immunohistochemical analysis, according to the manufacturer's protocol. Briefly, paraffin sections were deparaffinized, rehydrated, submerged in $3 \%$ hydrogen peroxide (Sinopharm Chemical Reagent Co., Ltd, Shanghai, China), washed with phosphate buffered saline and blocked with goat serum (Beijing Zongshan Golden Bridge Biotechnology Co., Ltd.). Then, the samples were incubated with rabbit polyclonal anti-TNF- $\alpha$ (1:500; bs 0078R), rabbit polyclonal anti IL-6 (1:500 dilution; bs6309R) and rabbit polyclonal anti IL-8 (1:500 dilution; bs0780R) (both purchased from Beijing Biosynthesis Biotechnology Co., Ltd.) overnight at $4^{\circ} \mathrm{C}$. Sections were then incubated with a biotinylated secondary antibody (polyclonal goat anti-rabbit IgG streptavidin antibody; 1:500 dilution; ZB-5301; Beijing Zhongshan Golden Bridge Biotechnology Co., Ltd.) at $37^{\circ} \mathrm{C}$ for $2 \mathrm{~h}$. The sections were then developed with diaminobenzidine and counterstained with hematoxylin and eosin (both purchased from Beyotime Institute of Biotechnology). The primary antibody was omitted in the negative control. Microscopic images were acquired using a Leica microscope (DM4000B; Leica Microsystems) and five high power fields (magnification, $\mathrm{x} 400$ ) were randomly selected in each slide. The average proportion of positive cells in each field were counted using Image Pro Plus (version 6.0; Media Cybernetics, Inc., Rockville, MD, USA). Immunohistochemical score was used to evaluate the slices $(24,25)$.

RNA extraction and reverse transcription-polymerase chain reaction (RT-PCR) analysis. Total RNA was extracted from the synovial tissues using TRIzol ${ }^{\circledR}$ (Invitrogen; Thermo Fisher Scientific, Inc., Waltham, MA, USA). Total RNA $(2 \mu \mathrm{g})$ was reverse transcribed to cDNA using a RevertAid First Strand cDNA Synthesis Kit (Thermo Fisher Scientific, Inc.). The reaction mixture contained $1 \mu$ l Oligo(dT)18 Primer, 5X Reaction Buffer, $1 \mu$ l Ribolock RNase Inhibitor (20 U/ $\mu 1), 2 \mu \mathrm{l}$ dNTP (10 mM), $1 \mu 1$ RevertAid M-MuLV Reverse Transcriptase (200 U/ $\mu \mathrm{l})$ and $20 \mu \mathrm{l}$ nuclease-free water. The obtained cDNA was used to determine the mRNA expression levels of IL-6, IL-8, TNF- $\alpha$ and NF- $\kappa$ B by PCR using Taq DNA polymerase (Thermo Fisher Scientific, Inc.). The PCR reaction volume was $20 \mu \mathrm{l}$, containing $10 \mu \mathrm{l}$ DreamTaq Green PCR Master Mix (2X), $0.4 \mu 1$ forward 
A

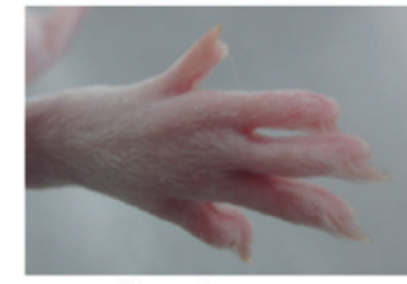

Normal group

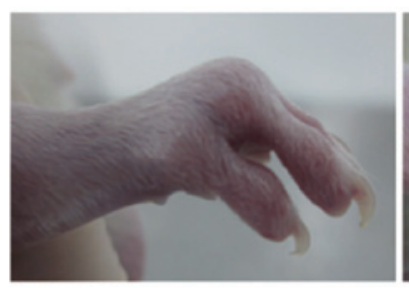

BCBE low group

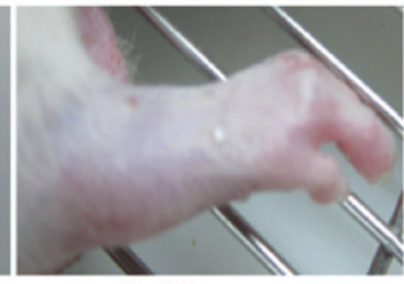

Model group

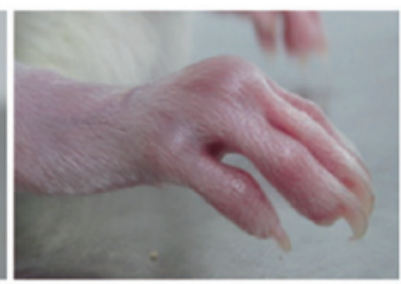

BCBE high group

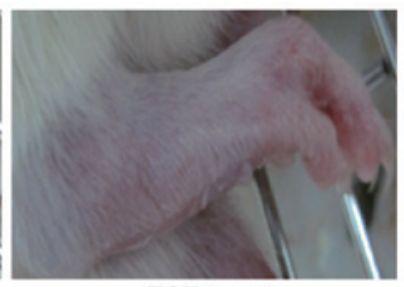

TGT group

B

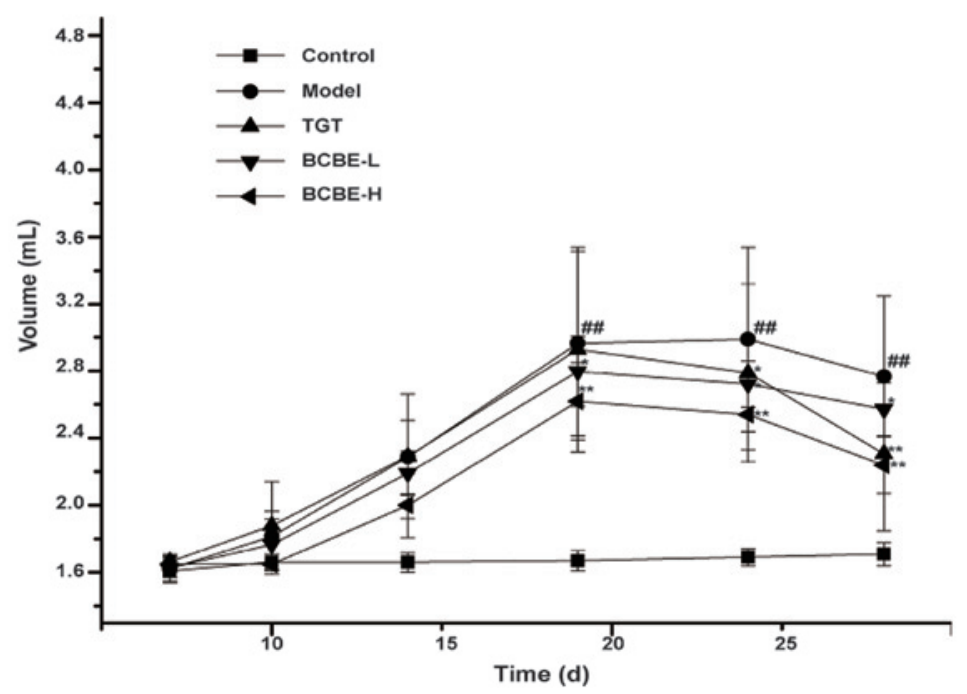

Figure 2. Right hind paw edema in the various rat groups. (A) Morphological characteristics of the right hind paw from the various rat groups. (B) Paw edema volume of the various rat groups. ${ }^{\# \#} \mathrm{P}<0.01$ vs. the control group; ${ }^{*} \mathrm{P}<0.05,{ }^{* *} \mathrm{P}<0.01$ vs. the model group. Data are presented as the mean \pm standard deviation. BCBE, Bauhinia championii (Benth.) Benth. extraction; TGT, tripterygium glycosides tablet.

primer $(1 \mu \mathrm{M}), 0.4 \mu 1$ reverse primer $(1 \mu \mathrm{M}), 1 \mu 1$ template DNA $(1 \mu \mathrm{g})$ and $8.2 \mu \mathrm{l}$ nuclease-free water. The PCR was conducted under the following conditions using a thermal cycler (C1000 Touch; Bio-Rad Laboratories, Inc., Hercules, CA, USA): Initial denaturation at $95^{\circ} \mathrm{C}$ for $3 \mathrm{~min}$, annealing at $95^{\circ} \mathrm{C}$ for $30 \mathrm{sec}$ and extension at $72^{\circ} \mathrm{C}$ for $45 \mathrm{sec}$. The annealing temperatures and number of amplification cycles were $50^{\circ} \mathrm{C}$ and 38 cycles for IL- $6,57^{\circ} \mathrm{C}$ and 35 cycles for IL- $8,58^{\circ} \mathrm{C}$ and 35 cycles for TNF- $\alpha, 60^{\circ} \mathrm{C}$ and 35 cycles for NF- $\mathrm{kB}$, and $55^{\circ} \mathrm{C}$ and 35 cycles for GAPDH. Primers were purchased Shanghai Sangon Biological Engineering Co., Ltd. (Shanghai, China), from and the sequences were as follows: IL-6, forward 5'-GAC AAAGCCAGAGTCCTTCA-3', reverse 5'-ACTAGGTTTGCC GAGTAGAC-3'; IL-8, forward 5'-GACTGTTGTGGCCCT GGAG-3', reverse 5'-CCGTCAAGCTCTGGATGTTCT-3'; TNF- $\alpha$, forward 5'-CTCCCAGGTTCTCTTCAAGG-3', reverse 5'-TGGAAGACTCCTCCCAGGTA-3'; NFforward 5'-GCGCATCCAGACCAACAATAAC-3', and reverse 5'-GCCGAAGCTGCATGGACACT-3'; and GAPDH, forward 5'-ACTGGCATTGTGATGGACTC-3', and reverse 5'-CAGCACTGTGTTGGCATAGA-3'. Glyceraldehyde 3-phosphate dehydrogenase was used as an internal control.
All processes were performed on the basis of manufacturer's protocol. Finally, the PCR products were analyzed by gel electrophoresis (1.5\% agarose), the agarose gels were stained using GoldView (Beyotime Institute of Biotechnology) and DNA bands were examined using a Gel Documentation system (Gel Doc 2000; Bio-Rad Laboratories, Inc.).

Statistical analysis. Data are presented as the mean \pm standard deviation. One-way analysis of variance and the Student-Newman-Keuls post-hoc test were used to determine significant differences between the groups. SPSS software (version 16.0; SPSS, Inc., Chicago, IL, USA) was used to perform statistical analysis. $\mathrm{P}<0.05$ was considered to indicate a statistically significant difference.

\section{Results}

Effects of BCBE on paw edema. The general condition of the rats was monitored during treatment. The onset time of paw edema for the majority of rats was day 3 after the second immunization. Post-treatment, red swelling in the right hind paw was markedly ameliorated in the BCBE-treated groups 
Table I. Serum levels of IL-6, IL-8, IL-10 and TNF- $\alpha$ in a rat model of type II collagen-induced arthritis in response to BCBE.

\begin{tabular}{lcccc}
\hline Group & IL-6 $(\mathrm{pg} / \mathrm{ml})$ & IL-8 $(\mathrm{ng} / \mathrm{ml})$ & $\mathrm{IL}-10(\mathrm{pg} / \mathrm{ml})$ & $\mathrm{TNF}-\alpha(\mathrm{ng} / \mathrm{ml})$ \\
\hline Normal & $65.958 \pm 2.582$ & $0.131 \pm 0.015$ & $49.403 \pm 1.744$ & $0.284 \pm 0.042^{\mathrm{a}}$ \\
Model & $160.854 \pm 3.221^{\mathrm{b}}$ & $0.296 \pm 0.013^{\mathrm{b}}$ & $35.792 \pm 2.681^{\mathrm{b}}$ & $1.079 \pm 0.087^{\mathrm{b}}$ \\
TGT & $86.281 \pm 3.677^{\mathrm{a}}$ & $0.232 \pm 0.035^{\mathrm{c}}$ & $40.854 \pm 1.461^{\mathrm{a}}$ & $0.646 \pm 0.038^{\mathrm{a}}$ \\
BCBE low & $97.513 \pm 3.355^{\mathrm{a}}$ & $0.262 \pm 0.041$ & $38.001 \pm 1.783$ & $0.897 \pm 0.074^{\mathrm{c}}$ \\
BCBE high & $81.363 \pm 3.468^{\mathrm{a}}$ & $0.186 \pm 0.050^{\mathrm{a}}$ & $39.412 \pm 1.988^{\mathrm{a}}$ & $0.469 \pm 0.091^{\mathrm{a}}$ \\
\hline
\end{tabular}

Data are presented as the mean \pm standard deviation. ${ }^{a} \mathrm{P}<0.01$, vs. the model group; ${ }^{\mathrm{b}} \mathrm{P}<0.01$, vs. the normal group; ${ }^{\mathrm{c}} \mathrm{P}<0.05$, vs. the model group. IL, interleukin; TNF- $\alpha$, tumor necrosis factor- $\alpha$; BCBE, Bauhinia championii (Benth.) Benth. extraction.

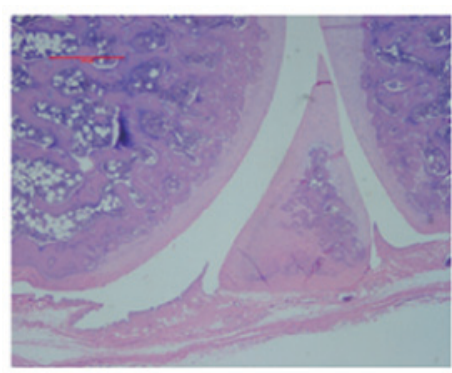

Normal group

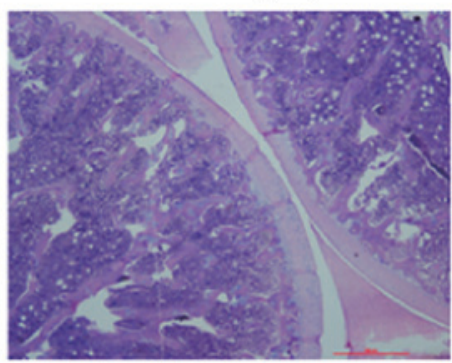

BCBE low group

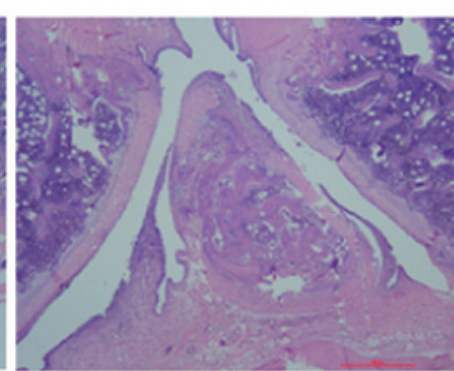

Model group

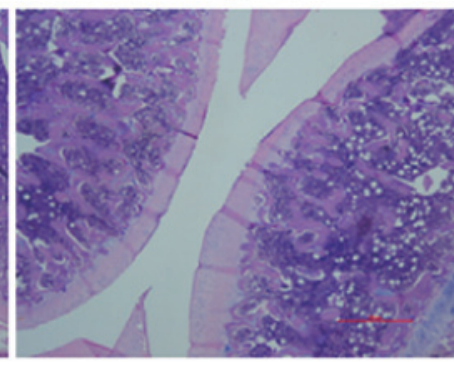

BCBE high group

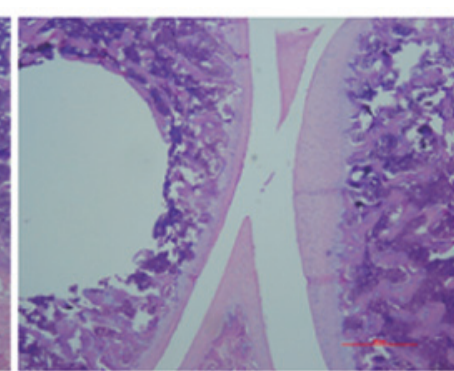

TGT group

Figure 3. Effects of Bauhinia championii (Benth.) Benth. extraction (BCBE) on the histopathology of rat ankle joints. Representative images are presented (hematoxylin and eosin; magnification, 4x). TGT, tripterygium glycosides tablet.

(Fig. 2A). As shown in Fig. 2B, paw edema was observed between days 1 and 28. Paw edema increased in severity over time, and paw swelling volume reached its maximum on day 18. However, paw edema in the BCBE groups increased more slowly compared with the model group, and was significantly decreased after day $18(\mathrm{P}<0.05)$. With therapeutic action, the paw swelling volume was gradually reduced over time. Furthermore, the edema-reducing effects of BCBE appeared earlier than those of TGT.

Effects of BCBE on serum cytokine levels in CIA rats. As shown in Table I, the serum levels of IL-6, IL- 8 and TNF- $\alpha$ were significantly increased in the model group compared with in the normal group $(\mathrm{P}<0.01)$, thus suggesting that the model group exhibited a marked inflammatory response. However, the cytokine levels were decreased in the TGT and BCBE groups. Furthermore, the levels of IL-6, IL-8 and TNF- $\alpha$ differed between the high-dose and model groups $(\mathrm{P}<0.01)$. These results suggest that $\mathrm{BCBE}$ may reduce the levels of proinflammatory cytokines, in order to dose-dependently relieve CIA-associated inflammation and gradually alleviate
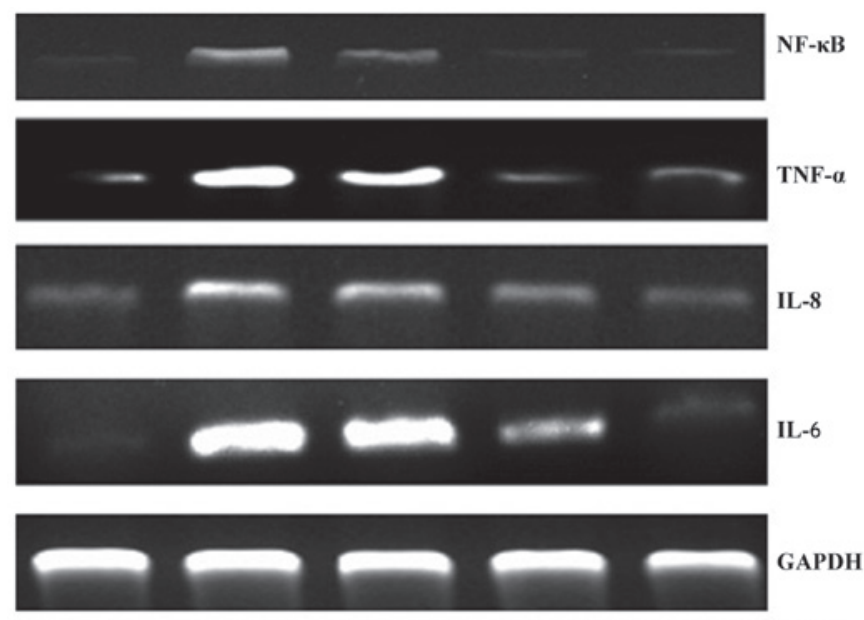

Normal group Model group TGT group BCBE low group BCBE high group

Figure 4. Effects of Bauhinia championii (Benth.) Benth. extraction (BCBE) on the mRNA expression levels of interleukin (IL)-6, IL-8, tumor necrosis factor (TNF)- $\alpha$ and nuclear factor (NF)-кB. The levels of IL-6, IL-8, TNF- $\alpha$ and $\mathrm{NF}-\kappa \mathrm{B}$ were determined in synovial tissues using reverse transcription-polymerase chain reaction. Glyceraldehyde 3-phosphate dehydrogenase (GAPDH) was used as an internal control. TGT, tripterygium glycosides tablet. 
A

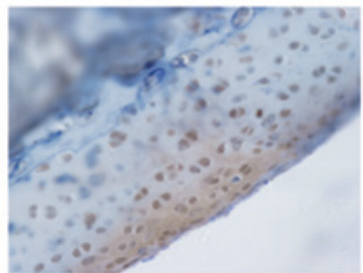

Normal group

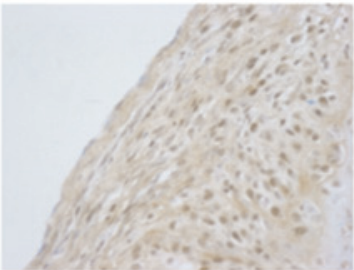

BCBE low group

$\mathbf{B}$

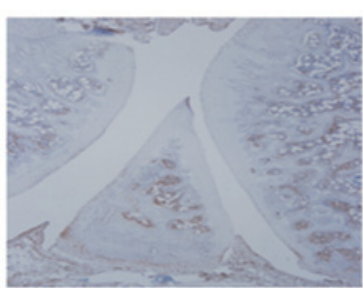

Normal group

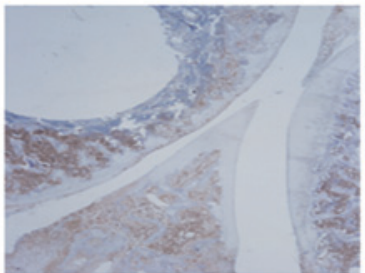

BCBE low group

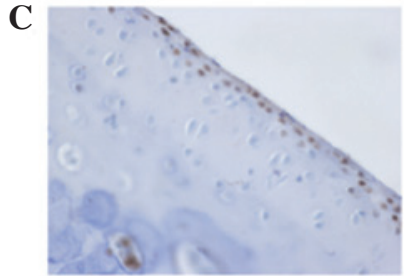

Normal group

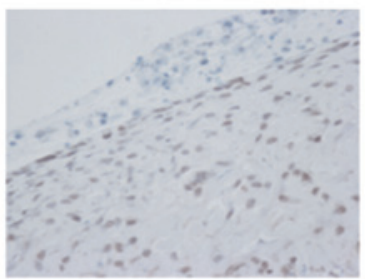

BCBE low group

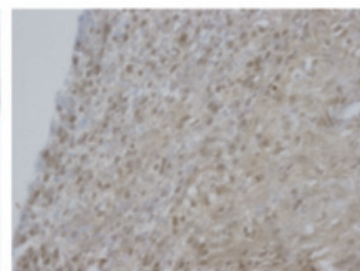

Model group

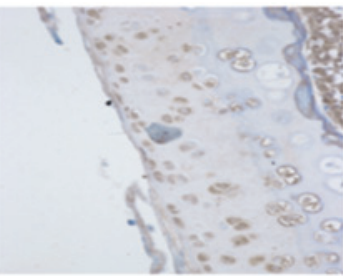

BCBE high group

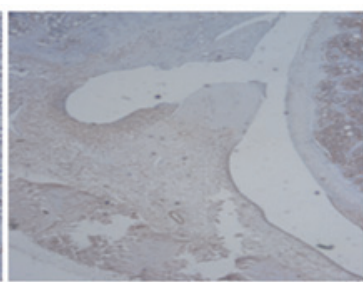

Model group

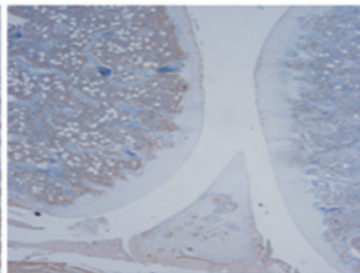

BCBE high group

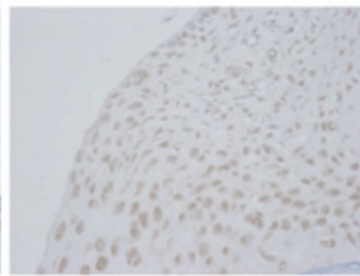

Model group

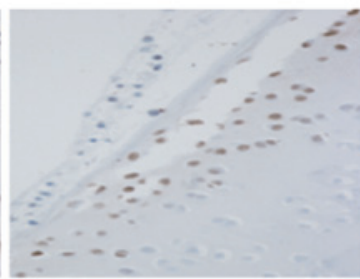

BCBE high group

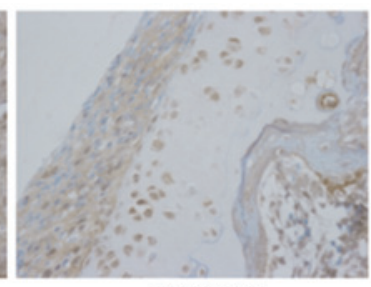

TGT group

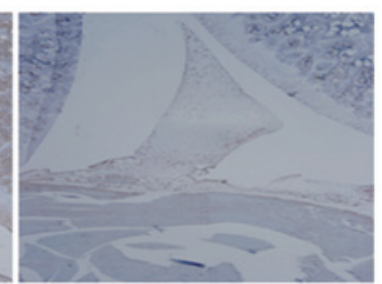

TGT group

D
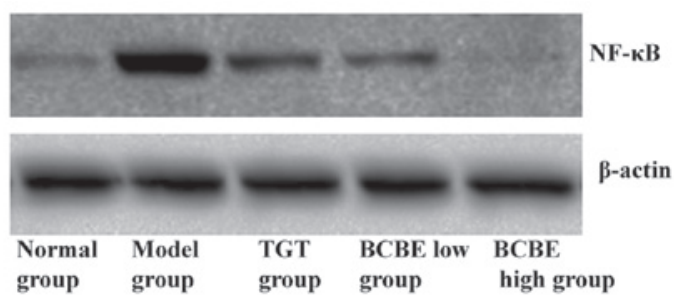

Figure 5. Effects of Bauhinia championii (Benth.) Benth. extraction (BCBE) on the expression of interleukin (IL)-6, IL-8, tumor necrosis factor (TNF)- $\alpha$ and nuclear factor (NF)- $\kappa$ B. The levels of (A) IL-6, (B) IL-8 and (C) TNF- $\alpha$ in synovial tissues were determined by immunohistochemistry (magnification $\mathrm{x} 40$ ), and (D) the levels of NF- $\mathrm{B}$ in synovial tissues were determined by western blotting. $\beta$-actin was used as a control. TGT, tripterygium glycosides tablet. 
joint damage. Conversely, the levels of the chemotactic cytokine IL-10 were markedly reduced in the model group, and were increased in the TGT and BCBE groups.

Effects of BCBE on histopathological alterations. As shown in Fig. 3, representative pathological sections from CIA rat joints were stained with HE. Rats in the normal group exhibited a normal histological architecture. Conversely, the model group exhibited markedly abnormal histological architecture, including significant synovial cell proliferation with a disorganized arrangement, hyperplasia, inflammation, and extensive erosive changes to cartilage and bone. The BCBE-treated rats exhibited a nearly normal histological architecture compared with the model group. In the BCBE low-dose group, synovial tissue displayed moderate hyperplasia and infiltration of inflammatory cells, with pannus formation and some cartilage damage. In the BCBE high-dose group, only mild synovial proliferation was observed, cell morphology was normal, a small number of inflammatory cells had infiltrated, no typical pannus had formed, and the cartilage surface was smooth with no obvious damage and no obvious bone erosion.

Effects of $B C B E$ on the expression levels of $I L-6, I L-8$, $T N F-\alpha$ and $N F-\kappa B$. The mRNA expression levels of IL-6, IL-8, TNF- $\alpha$ and NF- $\kappa$ B are presented in Fig. 4. In the model group, the mRNA expression levels of IL-6, IL-8, TNF- $\alpha$ and $\mathrm{NF}-\kappa \mathrm{B}$ were increased compared with those in the normal group; however, the expression levels were reduced following treatment with TGT and different doses of BCBE. Results from immunohistochemistry and western blotting indicated that the patterns of IL- $6, \mathrm{IL}-8, \mathrm{TNF}-\alpha$ and NF- $\kappa \mathrm{B}$ protein expression were similar to their respective mRNA expression patterns (Fig. 5A-D).

\section{Discussion}

It is well-known that CIA is similar to human RA with regards to clinical manifestations, serological parameters, and immunological and pathological alterations. In the present study, CIA was established as an experimental animal model of RA. The general condition of the rats was monitored and paw edema was observed, suggesting the model was successful, since arthritis is characterized by paw redness and paw edema. In addition, according to histopathological analysis of the ankle joint, anomalous hyperplasia of synovial membranes, collagen fiber deposition, large numbers of inflammatory cells, and cartilage and bone erosion were detected in the model group. These phenomena were also described in previous studies $(26,27)$, and were attenuated following treatment with $\mathrm{BCBE}$. Therefore, BCBE may relieve paw edema and alleviate synovial membrane hyperplasia, thus indicating that $\mathrm{BCBE}$ may possess certain anti-inflammatory effects.

TNF- $\alpha$ is an important inflammatory mediator that can stimulate the secretion of numerous other inflammatory cytokines. It has been reported that the main pathological features of RA are closely associated with immunomodulatory cytokines, including proinflammatory cytokines (TNF- $\alpha$, IL-6 and IL-8) and anti-inflammatory cytokines (IL-10) in serum $(28,29)$. Previous studies have demonstrated that
TNF- $\alpha$ overproduction is associated with the progression of RA, thus suggesting that it may be used as a parameter for the evaluation of disease-modifying anti-rheumatic drugs $(30,31)$. The present study indicated that the levels of proinflammatory cytokines (TNF- $\alpha$, IL- 6 and IL-8) were substantially increased in the model group, whereas IL-10 was decreased. Furthermore, the treatment groups exhibited marked suppression of TNF- $\alpha$, IL- 6 and IL- 8 expression, whereas IL-10 expression was increased. These results suggested that $\mathrm{BCBE}$ was effective at suppressing the development of proinflammatory cytokines, but increased the expression of IL-10. Therefore, BCBE may be considered a potential therapeutic or preventive candidate for the treatment of acute inflammation in RA.

$\mathrm{NF}-\kappa \mathrm{B}$ is a pleiotropic transcription factor and a pivotal regulator of RA-associated inflammation. It is inactive in quiescent cells, but once activated, it can promote the transcription of several genes and release cytokines that contribute to inflammation. $\mathrm{NF}-\kappa \mathrm{B}$ is essential for triggering and amplifying the cytokine network (32). It has previously been reported that the $\mathrm{NF}-\kappa \mathrm{B}$ signaling pathway may result in downregulation of the expression of various anti-apoptotic and cytokine genes (33). In addition, $N F-\kappa B$ has been demonstrated to regulate the expression of TNF- $\alpha$ and IL-6 (34). As determined by western blot analysis, NF- $\kappa \mathrm{B}$ was significantly inhibited in the synovial tissue by BCBE. In conclusion, the anti-inflammatory effects of BCBE may be associated with the inhibition of NF- $\kappa \mathrm{B}, \mathrm{TNF}-\alpha, \mathrm{IL}-6$ and IL-8, and the induction of IL-10; however, further evidence is required.

\section{Acknowledgements}

The present study was performed in the State Key Laboratory of Chinese Pharmacies of Fujian Provincial Department of Science and Technology, Collaborative Innovation Center for Rehabilitation Technology and Traditional Chinese Medicine Rehabilitation Research Center of the State Administration of Traditional Chinese Medicine. The present study was financially supported by the National Natural Science Foundation of China (grant no. 81370096), Fujian Province Science-Technology Plan Projects (grant no. 2010Y2004) and the 2014 National College Students' Innovative Entrepreneurial Training Plan Project (grant no. 201410393008).

\section{References}

1. Brenner M, Meng HC, Yarlett NC, Joe B, Griffiths MM, Remmers EF, Wilder RL and Gulko PS: The non-MHC quantitative trait locus Cia5 contains three major arthritis genes that differentially regulate disease severity, pannus formation, and joint damage in collagen- and pristane-induced arthritis. J Immunol 174: 7894-7903, 2005.

2. Ono Y, Inoue M, Mizukami H and Ogihara Y: Suppressive effect of Kanzo-bushi-to, a Kampo medicine, on collagen-induced arthritis. Biol Pharm Bull 27: 1406-1413, 2004.

3. Helliwell P, Woodburn J, Redmond A, Turner D and Davys H (eds.): Current concepts in rheumatoid arthritis. In: The Foot and Ankle in Rheumatoid Arthritis: A Comprehensive Guide. Elsevier Health Sciences, Edinburgh, pp1-16, 2007.

4. Kochi Y, Suzuki A, Yamada R and Yamamoto K: Genetics of rheumatoid arthritis: Underlying evidence of ethnic differences. J Autoimmun 32: 158-162, 2009. 
5. Symmons DP: Environmental factors and the outcome of rheumatoid arthritis. Best Pract Res Clin Rheumatol 17: 717-727, 2003

6. Huizinga TW: Genetics in rheumatoid arthritis. Best Pract Res Clin Rheumatol 17: 703-716, 2003.

7. Kallberg H, Padyukov L, Plenge RM, Ronnelid J, Gregersen PK, van der Helm-van Mil AH, Toes RE, Huizinga TW, Klareskog L and Alfredsson L; Epidemiological Investigation of Rheumatoid Arthritis study group: Gene-gene and gene-environment interactions involving HLA-DRB1, PTPN22, and smoking in two subsets of rheumatoid arthritis. Am J Hum Genet 80: $867-875,2007$

8. Mackenzie AR and Dawson J: Could rheumatoid arthritis have an infectious aetiology? Drug Discovery Today: Disease Mechanisms 2: 345-349, 2005.

9. De Roos AJ, Cooper GS, Alavanja MC and Sandler DP: Rheumatoid arthritis among women in the Agricultural Health Study: Risk associated with farming activities and exposures. Ann Epidemiol 15: 762-770, 2005.

10. Davis JM III and Matteson EL: Cytokine biomarkers and the promise of personalized therapy in rheumatoid arthritis. Reumatol Clin 5: 143-146, 2009 (In Spanish).

11. van den Berg WB: Anti-cytokine therapy in chronic destructive arthritis. Arthritis Res 3: 18-26, 2001

12. Jazayeri JA, Carroll GJ and Vernallis AB: Interleukin-6 subfamily cytokines and rheumatoid arthritis: Role of antagonists. Int Immunopharmacol 10: 1-8, 2010.

13. Brennan FM, Zachariae CO, Chantry D, Larsen CG, Turner M, Maini RN, Matsushima K and Feldmann M: Detection of interleukin 8 biological activity in synovial fluids from patients with rheumatoid arthritis and production of interleukin $8 \mathrm{mRNA}$ by isolated synovial cells. Eur JImmunol 20: 2141-2144, 1990.

14. Persson S, Mikulowska A, Narula S, O'Garra A and Holmdahl R: Interleukin-10 suppresses the development of collagen type II-induced arthritis and ameliorates sustained arthritis in rats. Scand J Immunol 44: 607-614, 1996.

15. Jiangsu New Medical College: Dictionary of Chinese Traditional Drugs, Shanghai Scientific and Technical Publishers: Shanghai, China: p43, 1986

16. Guo CQ and Xiang RM: Treatment of acute or chronic backleg pain by yangtiteng. Minzu Minjian Yiyao 9: 61, 2001 (In Chinese).

17. Wei HS and Qi ZY: Curative effect observation of fumigation treatment arthralgia 58 cases. Zhongyi Waizhi Zazhi 7: 41-42, 1998 (In Chinese).

18. Xie GC and Xiao QQ (eds.): Chinese Illuminations of Well-Triedrecipe of Herbs. Shantou University Press, Shantou, China, p125, 2000.

19. Fang D, Luo JY, Su GX, Tao YP, Tan XY and Tan DH: Selectional medications prescription of Zhuang folk medicine. Guangxi Nationalities Publishing House, Guangxi, China, pp6-77, 1985.

20. Zheng H, Xu W, Li H, Chu K, Chen L and Zhang Y: Effects of ethyl acetate extracts from Bauhinia championii (Benth.) Benth. on rats with collagen-induced arthritis. Fu Jian Zhong Yi Xue Yuan Xue Bao 23: 31-34, 2013.
21. Institute of Laboratory Animal Resources (US). Committee on Care, Use of Laboratory Animals, and National Institutes of Health (US). Division of Research Resources: Guide for the care and use of laboratory animals. 8th edition. National Academies Press, Washington, DC, 2011.

22. Courtenay JS, Dallman MJ, Dayan AD, Martin A and Mosedale B: Immunization against heterologous type II collagen induces arthritis in mice. Nature 283: 666-668, 1980.

23. Gao Q, Shan J, Di L, Jiang L and Xu H: Therapeutic effects of daphnetin on adjuvant-induced arthritic rats. J Ethnopharmacol 120: 259-263, 2008.

24. Chen Q and Wei W: Effects and mechanisms of glucosides of chaenomeles speciosa on collagen-induced arthritis in rats. Int Immunopharmacol 3: 593-608, 2003.

25. Brand DD, Kang AH and Rosloniec EF: Immunopathogenesis of collagen arthritis. Springer Semin Immunopathol 25: 3-18, 2003.

26. Keyszer G, Redlich A, Häupl T, Zacher J, Sparmann M, Engethüm U, Gay S and Burmester GR: Differential expression of cathepsins B and L compared with matrix metalloproteinases and their respective inhibitors in rheumatoid arthritis and osteoarthritis: A parallel investigation by semiquantitative reverse transcriptase-polymerase chain reaction and immunohistochemistry. Arthritis Rheum 41: 1378-1387, 1998.

27. Myers LK, Rosloniec EF, Cremer MA and Kang AH: Collagen-induced arthritis, an animal control of autoimmunity. Life Sci 61: 1861-1878, 1997.

28. Brennan FM, Chantry D, Jackson A, Maini R and Feldmann M Inhibitory effect of TNF alpha antibodies on synovial cell interleukin-1 production in rheumatoid arthritis. Lancet 2: 244-247, 1989

29. Tanida S, Yoshitomi H, Nishitani K, Ishikawa M, Kitaori T, Ito H and Nakamura T: CCL20 produced in the cytokine network of rheumatoid arthritis recruits $\mathrm{CCR}^{+}$mononuclear cells and enhances the production of IL-6. Cytokine 47: 112-118, 2009.

30. DiMartino M, Wolff C, High W, Stroup G, Hoffman S, Laydon J, Lee JC, Bertolini D, Galloway WA, Crimmin MJ, et al: Anti-arthritic activity of hydroxamic acid-based pseudopeptide inhibitors of matrix metalloproteinases and TNF alpha processing. Inflamm Res 46: 211-215, 1997.

31. Hisadome M, Fukuda T, Sumichika H, Hanano T and Adachi K: A novel anti-rheumatic drug suppresses tumor necrosis factor-alpha and augments interleukin-10 in adjuvant arthritic rats. Eur J Pharmacol 409: 331-335, 2000.

32. Morel J and Berenbaum F: Signal transduction pathways: New targets for treating rheumatoid arthritis. Joint Bone Spine 71: 503-510, 2004.

33. Osorio Y Fortéa J, Bukulmez H, Petit-Teixeira E, Michou L, Pierlot C, Cailleau-Moindrault S, Lemaire I, Lasbleiz S, Alibert O, Quillet $\mathrm{P}$, et al: Dense genome-wide linkage analysis of rheumatoid arthritis, including covariates. Arthritis Rheum 50: 2757-2765, 2004.

34. Tu S, Hu Y, Zeng K, Zhang M, Lai X and Weichen Z: Effects of triptolide on the expression and activity of NF-kappaB in synovium of collagen induced arthritis rats. J Huazhong Univ Sci Technolog Med Sci 25: 543-545, 2005. 